\title{
Silane-Bridged Electroless Ni-Plating on Submicron Polymer Spheres
}

\author{
By Wen-Chang LIAW, ${ }^{1, *}$ Po-Chen HUANG, ${ }^{1}$ Kuan-Pin CHEN, ${ }^{1}$ and Chee-Shan CHEN $^{2}$
}

The silane coupling agent (3-trimethoxysilyl propryl methacrylate) was used to functionalize the surface of submicron polymer spheres (sized 168-650 nm) for bridging the polymer core (polystyrene crosslinked with divinylbenzene) and the metal coating shell. The submicron polymer spheres were synthesized by emulsifier-free emulsion polymerization in the presence of $\mathrm{NaOH}$. The metal shell was created by electroless plating. The performance of silane coupling agent, as the bondage between the core and the shell, was compared, in terms of thermal, mechanical and electrical properties, to that of polymer spheres functionalized with - $\mathrm{COOH}$ groups. The silane bridge provided more opportunities for binding with metal and the bondage is stable, leading to a denser and more uniform distribution of metal coating, as observed by FESEM studies. The silane bridge was proven to be more durable than the case of $-\mathrm{COOH}$ by ball mill tests. Besides bridging with the metal, neighboring silyl groups also form Si-O-Si 2-dimensional network by condensation between two Si-OHs, which further stabilized the bondage between the polymer core and the metal shell.

KEY WORDS: Silane Coupling Agent / Submicron Polymer Sphere / Electroless Plating / Core-Shell Structure / Ni Plating /

A polymeric microsphere coated with a metallic film (similar to a core-shell structure) would exhibit good electrical conductivity. Due to its spherical geometry, and meanwhile, the major weight portion of the electric-conducting microsphere is occupied by the polymer core, the material has physical properties (e.g., density), as well as price, that are different from metal particles of similar size.

In recent years, considerable efforts have been directed to the design and selection of fabrication method for the core polymer microspheres' preparation and metal film coating to obtain the metal coated polymer microspheres with specific electrical, mechanical and chemical properties. Such polymeric microspheres with metallic surfaces have potential applications in interface science, heterogeneous catalysis, electronics etc. ${ }^{1-3}$

In the literature, a few different approaches have been developed to produce uniformly sized core polymer microsphere colloid, such as suspension polymerization, ${ }^{1,4}$ emulsion polymerization, ${ }^{5}$ and dispersion polymerization. ${ }^{6-9}$ Although by traditional emulsion polymerization, the diameters of the core polymer microspheres could be reduced to submicron scale, but the size distribution could undesirably wide. Better control over the size distribution of the submicron polymer cores calls for the need of alternative strategy. ${ }^{2,5}$ Zhang et al. ${ }^{10}$ demonstrated that the emulsifier-free emulsion polymerization is a process that is capable of obtaining submicron scaled polymer spheres with narrow distribution of particle size. They also pointed out that the merit of such process is the spherical geometry of the product. $^{10}$

The method of metallic coating is another technological issue in fabricating such material. Metallic coating can be achieved by direct surface reactions of the specific functional groups on the polymer core with vaporized metal in physical vapor deposition (PVD). This method suffers high processing costs of the PVD system. ${ }^{11,12}$ Although it will be interesting to compare costs and qualities of end product, such comparison would be practical only after available technologies have been thoroughly studied.

In addition to PVD metal plating at the vapor-solid interface, chemical electroless plating (at the liquid-solid interface) is another alternative for metallic coating, ${ }^{13-15}$ in which, a metal ion is reduced with a reducing agent in the plating solution and deposited, in its elemental form, on the surface of the polymer substrate. To start and accelerate the reduction reaction on the surface of a polymer substrate, and to improve the adhesion of the metallic coating, the polymer surface must first be sensitized by tin chloride ${ }^{16}$ right after cleansing and etching of the substrate. The sensitized surface can then be activated by a noble metal ion such as palladium chloride. Finally, another metal (e.g., $\mathrm{Ni}, \mathrm{Au}, \mathrm{Co}, \mathrm{Cu}$, and $\mathrm{Ag}$ ) can be deposited on the sensitized and activated polymer surface by chemical electroless plating. Guo et al. ${ }^{3}$ prepared a micron sized nickel-phosphorus alloy coated polymethyl methacrylate (PMMA) particles by chemical electroless plating technique. The prepared particles consist of a PMMA core inside a shell of nickel-phosphorus alloy. The obtained particles exhibit magnetism and can be aligned to form a long chain structure under applied magnetic field. ${ }^{3}$

More recently, the noble metal (e.g., Pd) activating process could be enhanced by introducing organic functional groups on the polymer surface. Then, the metal ion was allowed to react with these functional groups, before being reduced to metallic state, to induce hybrid (organic functional group/metal ion)

\footnotetext{
${ }_{1}^{1}$ Department of Chemical and Materials Engineering, National Yunlin University of Science and Technology, 123, Section 3, University Road, Touliu, Yunlin 640, Taiwan

${ }^{2}$ Department of Applied Chemistry, Chaoyang University of Technology, 168 Gifeng E. Road, Wufeng, Taichung 413, Taiwan

*To whom correspondence should be addressed (Tel: +886-5-5342601-4618, Fax: +886-5-5312071, E-mail: Liawwc@ yuntech.edu.tw).
} 
metal coating. ${ }^{6,17}$ Candidate polymer functional groups such as amino, carboxylic and thiol have been reported, they generally exhibit nucleophilic character. Warshawsky and Upson ${ }^{17}$ reported the mechanism of the electroless plating on functionalized polymer particles of various sizes. The functional groups (amino, carboxylic or thiol) on the surface of polymers served as catalytic sites for activation through binding of noble metal ions by coordination or ionic bonding, followed by chemical reduction of the metal ion to zerovalent metal state. ${ }^{17}$

Jun et ll $^{6}$ synthesized amino group functionalized polymer microspheres by emulsion polymerization of styrene ( $\mathrm{St}$ ). In the process, divinylbenzene (DVB) served as the crosslinking agent, and glycidyl methacrylate (GMA) was used to prepare for later function ligand establishment on the polymer surface. The glycidyl group on the obtained microspheres was allowed to react with ethylene diamine to produce aminoethyl group on the surface of the polymer microspheres (with another amino group available for binding with metal). Such free and exposed amino groups on the surface of the polymer microspheres facilitated subsequent chemical electroless plating. ${ }^{6}$

Park et $a l .{ }^{18}$ studied the effects of different surface functionality on nickel deposition. They successfully produced micron sized metal coating on polymer microspheres functionalized with the thiol group.

In the above mentioned function group facilitated metal coating on the micron or submicron polymer particles, the functional groups played the role of bridging the organic (polymer) and the inorganic (metal) matrices. Although it remains to be seen if the trialkoxysilyl group of silane coupling agent would serve the same purpose as the aforementioned functional groups, or even improve the material in certain ways, it would be an interesting topic to investigate.

The silane coupling agent has a trialkoxysilyl group, wich is capable of reacting with the hydroxyl groups on the surface of metal and leads to the formation of Metal-O-Si (M-O-Si) covalent bond. ${ }^{19-21}$ Theoretically such covalent bond formation at the interface of organic-inorganic matrices is quite likely to increase the adhesion between the two. The use of such type of $\mathrm{M}-\mathrm{O}-\mathrm{Si}$ organic-inorganic covalent bonding has been successful in the above cited literatures.

For metal-on-polymer coatings, the same principle should apply, but the reversed process may lead to different results. Strategically, if the acrylic function group of the silane coupling agent was first allowed to react with crosslinked polystryrene with the trialkoxysilyl or trihydroxysilyl group exposed on the polymer surface, the latter would be available for the formation of Si-O-Metal (Si-O-M) covalent bonding during metal deposition. However, such optimistic anticipation may be clouded by possible condensation reaction between coupling agent molecules and lead to loss of stability of the emulsified polymer core and/or the adhesion between the organic core and the inorganic metal coating.

The main purpose of this research is to study the use of silane coupling agent as a covalent bridge between the submicron polymer spheres and the metal layer on the surface. The chemical structure, morphology and contribution of such covalent bridging between the metal and the polymer matrices are also major concerns of this study.

\section{EXPERIMENTAL}

\section{Materials}

Styrene (St; chemical reagent, Fluka, Milwaukee, WI) was distilled at $40^{\circ} \mathrm{C} / 14.5 \mathrm{mmHg}$ and stored at $4{ }^{\circ} \mathrm{C}$. Divinylbenezne (DVB, chemical reagent, Fluka, Milwaukee, WI) was distilled at $40^{\circ} \mathrm{C} / 12 \mathrm{mmHg}$, Acrylic acid (AA, chemical reagent, Fluka, Milwaukee, WI) was distilled at $40^{\circ} \mathrm{C} /$ $12 \mathrm{mmHg}$. Methacrylic acid (MAA, chemical reagent, Fluka, Milwaukee, WI) was distilled at $40^{\circ} \mathrm{C} / 12 \mathrm{mmHg}$, 3-trimethoxysilyl propryl methacrylate (TSPM, silane coupling agent, was obtained from Shin-Etsu silicone Taiwan Co., LTD., Taipei, Taiwan), were used in the synthesis without purification. Potassium persulfate $\left(\mathrm{K}_{2} \mathrm{~S}_{2} \mathrm{O}_{8}, 99.99 \%\right.$, initiator, Fluka, Milwaukee, WI) was recrystallized in deionized water before use. tin chloride dihydrate $\left(\mathrm{SnCl}_{2} \cdot 2 \mathrm{H}_{2} \mathrm{O}\right.$, chemical reagent, Fluka, Milwaukee, WI), palladium chloride $\left(\mathrm{PdCl}_{2}\right.$, chemical reagent, Dahao Chemical Plant of Guangdong, Guangdong, China), nickel chloride hexahydrate $\left(\mathrm{NiCl}_{2} \cdot 6 \mathrm{H}_{2} \mathrm{O}\right.$, chemical reagent, Fluka, Milwaukee, WI), and sodium hypophosphite dihydrate $\left(\mathrm{NaH}_{2} \mathrm{PO}_{2} \cdot 2 \mathrm{H}_{2} \mathrm{O}\right.$, chemical reagent, Taicang Second Chemical Plant of Jiangsu, Jangsu, China) were used without further purification. Deionized water was used in all experiments.

\section{Preparation of Functionalized Submicron Polymer Sphere}

A typical recipe for BA-1 to BA-5, BM-1 to BM-5, BT-1 to BT-5 microspheres is shown in Table I. The polymerization temperature was fixed at $70{ }^{\circ} \mathrm{C}$, and the speed of the stirrer was $200 \mathrm{rpm}$ in a nitrogen atmosphere to eliminate oxygen effects. Total conversion was determined gravimetrically. Polymerization was carried out in a $1000 \mathrm{~mL}$ five-neck round-bottom flask equipped with stirrer, reflux condenser, thermometer, nitrogen inlet tube, and feeding funnel. A small portion of monomer ( $\mathrm{St}$ and DVB; functional monomers AA, MAA or TSPM) and initiator $\left(\mathrm{K}_{2} \mathrm{~S}_{2} \mathrm{O}_{8}\right)$ were added first. After $1 \mathrm{~h}$, the rest of the monomer was added gradually (over $2.5 \mathrm{~h}$ ), through a peristaltic pump, to the flask. After another $0.5 \mathrm{~h}$, the rest of the initiator was then added to the flask. The reacting system was stirred for another $3 \mathrm{~h}$ to complete the polymerization (the conversion was $98 \%$ ).

Table I. Compositions of the core-shell Ni-plating submicron polymer spheres

\begin{tabular}{ccccccc}
\hline \multicolumn{2}{c}{ Sample Code } & $\begin{array}{c}\text { St } \\
\text { Before Ni-plating }\end{array}$ & $\begin{array}{c}\text { DVB } \\
\text { After Ni-plating }\end{array}$ & $\begin{array}{c}\text { AA } \\
(\%)\end{array}$ & $\begin{array}{c}\text { MAA } \\
(\%)\end{array}$ & $\begin{array}{c}\text { TSPM } \\
(\%)\end{array}$ \\
\cline { 1 - 5 } BC-0 & NiBC-0 & 97 & 3 & - & - & - \\
BT-1 & NiBT-1 & 96 & 3 & - & - & 1 \\
BT-2 & NiBT-2 & 95 & 3 & - & - & 2 \\
BT-3 & NiBT-3 & 94 & 3 & - & - & 3 \\
BT-4 & NiBT-4 & 93 & 3 & - & - & 4 \\
BT-5 & NiBT-5 & 92 & 3 & - & - & 5 \\
BA-3 & NiBA-3 & 94 & 3 & 3 & - & - \\
BM-3 & NiBM-3 & 94 & 3 & - & 3 & - \\
\hline
\end{tabular}




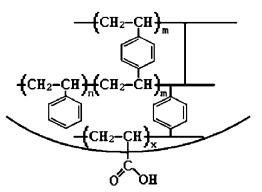

(a) P(St-DVB-AA)

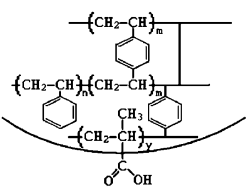

(b) P(St-DVB-MAA)

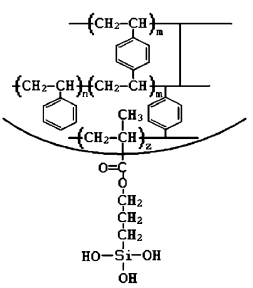

(c) P(St-DVB-TSPM)
Figure 1. Schematic diagram of the chemical structures of the polymer emulsions: (a) P(St-DVB-AA); (b) P(St-DVB-MAA); (c) P(St-DVBTSPM).

\section{Electroless Chemical Deposition on Submicron Polymer Spheres}

Nickel was coated onto BA, BM, BT polymer beads by electroless plating. The procedure is illustrated in Figure 1. A measured amount of submicron polymer sphere cores were first "sensitized" in acidic tin chloride $\left(\mathrm{SnCl}_{2}\right)$ solution with ultrasonic agitation for $1 \mathrm{~h}$. This would result in adsorption of $\mathrm{Sn}^{2+}$ ions onto the surface of the submicron polymer sphere cores. Then the submicron polymer sphere cores were washed with deionized water, to remove excessive $\mathrm{Sn}^{2+}$ ions.

The sensitized submicron polymer spheres were then suspended in a palladium chloride $\left(\mathrm{PdCl}_{2}\right)$ /hydrochloric acid solution for "surface activation." A surface redox reaction occurred in this step, which involved reduction of $\mathrm{Pd}^{2+}$ to $\mathrm{Pd}$ and oxidation of $\mathrm{Sn}^{2+}$ to $\mathrm{Sn}^{4+}$. In this step, metallic $\mathrm{Pd}$, serving as the catalytic sites, was formed which was chemically bounded to the functional groups (carboxyl or silyl) on the functionalized surface of the polymer spheres. The Pdactivated submicron polymer spheres were rinsed with deionized water, and then suspended in an electroless plating solution. The composition of the plating solution and the reaction condition are shown in Table I and II.

During chemical electroless plating, nickel ions were first reduced and formed nuclei on the Pd activated surfaces. The deposition process will continue automatically after the catalytic sites are plated with nickel because it is a selfcatalyzed reaction. Finally, the product was collected by placing a magnet at the bottom of the bath and washed twice with deionized water.

\section{Characterization}

The functional group of the polymer was verified by Fourier Transform Infrared (FT-IR) absorption spectra between 400 and $4000 \mathrm{~cm}^{-1}$ (Bruker ISF 112V FT-IR spectrometer, Germany). The thermal stability of the nanocomposites was characterized with a thermogravimetric analyzer (TGA), on a Perkin-Elmer TGA in $\mathrm{N}_{2}$ at a heating rate of $20^{\circ} \mathrm{C} \mathrm{min}^{-1}$ from 25 to $900{ }^{\circ} \mathrm{C}$. SEM images of the core/shell particles were taken on Jeol Model JSF 6700F field emission scanning electron microscope (Tokyo, Japan) which also provides energy-dispersion X-ray analysis (EDX).

The obtained core shell nickel plated submicron polymer spheres (CSNP spheres) was also investigated by transmission electron microscopy (TEM) with a JEOL-200 FX (Tokyo,
Table II. Compositions of the solutions for sensitization, activation and electroless plating

\begin{tabular}{cccc}
\hline \multirow{2}{*}{ Process } & Chemicals & \multicolumn{2}{c}{ Concentration (M) } \\
\cline { 3 - 4 } & & ENP-1 & ENP-2 \\
\hline \multirow{2}{*}{ Sensitization } & $\mathrm{SnCl}_{2} \cdot 2 \mathrm{H}_{2} \mathrm{O}$ & 0.044 & 0.044 \\
& $\mathrm{HCl}$ & 0.275 & 0.275 \\
\hline \multirow{2}{*}{ Activation } & $\mathrm{PdCl}_{2}$ & $5.6 \times 10^{-4}$ & $5.6 \times 10^{-4}$ \\
& $\mathrm{HCl}$ & 0.22 & 0.22 \\
\hline \multirow{2}{*}{ Elctroless Ni-Plating } & $\mathrm{NiCl}_{2} \cdot 6 \mathrm{H}_{2} \mathrm{O}$ & 0.033 & 0.066 \\
& $\mathrm{NaH}_{2} \mathrm{PO}_{2} \cdot \mathrm{H}_{2} \mathrm{O}$ & 0.02 & 0.04 \\
\hline
\end{tabular}

Japan) operating at $120 \mathrm{KV}$. Metal elements contained in the coated metal shell was identified by studying the X-ray diffraction (XRD) patterns with a Bruker P4 single crystal Xray diffractometer (Germany) at a scanning rate of $5^{\circ} \mathrm{min}^{-1}$ within the range of $2 \theta=20-80^{\circ}$. The adhesion between the coated metal layers and the polymer sphere was examined by ball milling for various length of time $(0.5,1,2,4$, and $8 \mathrm{~h})$, with a UBM-1 ball mill (Shin Kwang Machinery Industry, Taipei, Taiwan) at a rate of $150 \mathrm{rpm}$. The volume of the ball mill was $250 \mathrm{~mL}$. The diameter of the balls used for milling was $1.62 \mathrm{~mm}$. Ball loading was $100 \mathrm{~g}$ for $2 \mathrm{~g} \mathrm{CSNP}$ spheres in $10 \mathrm{~mL}$ water. The performances of the CSNP spheres as an electric conducting material in liquid suspension before and after ball mill treatment were compared in terms of the suspensions' conductivities measured with a conductivity meter (WM-221P conductimeter, East Asia Airwaves company, Tokyo, Japan).

\section{RESULTS AND DISCUSSIONS}

The silane-bridged electroless Ni-plated submicron polymer spheres were prepared as described in the experimental section. The core-shell nature and the contribution of the silane coupling agent, as well as various physical analyses are discussed in the following sections.

\section{Preparation of the Submicron Polymer Spheres}

In order to study the role of the coupling agent currently investigated as compared to that of $-\mathrm{COOH}$ ligand which has been successfully used in the literature, ${ }^{2}$ the carboxyl group $(\mathrm{COOH})$ functionalized BA-3 and BM-3 submicron polymer spheres were also prepared by functionalizing with acrylic acid (AA) or methacrylic acid (MAA) in place of TSPM in the emulsion polymerization (Figure 1).

Sample code of "BC-0" was the submicron polymer spheres without functionalization. Functionalized submicron polymer spheres were coded as BT-1 to BT-5, BA-3 and BM-3 (content of functional monomers ranged from 1 to $5 \mathrm{wt} \%$ ). As shown in Table III, when the content of functional monomer increased from 1 to $5 \mathrm{wt} \%$, the particle size, measured by light scattering, increased from approximately 168 to $650 \mathrm{~nm}$. They are thus called submicron polymer spheres.

Warshawsky and Upson ${ }^{17}$ compared various functional ligands for binding a noble metal (Pd) for the step of surface activation. These bound Pd metal then served as catalytic sites 
Table III. Particle sizes before and after electroless Ni-plating

\begin{tabular}{cccc}
\hline \multicolumn{2}{c}{ Sample Code } & \multicolumn{2}{c}{ Particle Size (nm) } \\
\hline Before Ni-plating & After Ni-plating & Before Ni-plating & After Ni-plating \\
\hline BC-0 & NiBC-0 & 280 & - \\
BT-1 & NiBT-1 & 235 & 279 \\
BT-2 & NiBT-2 & 270 & 327 \\
BT-3 & NiBT-3 & 282 & 335 \\
BT-4 & NiBT-4 & 388 & 458 \\
BT-5 & NiBT-5 & 650 & 796 \\
BA-3 & NiBA-3 & 168 & 233 \\
BM-3 & NiBM-3 & 248 & 311 \\
\hline
\end{tabular}

for deposition of a transition metal in the electroless chemical plating. In their design, the functional ligands were able to form complexes with Pd through coordination bonding.

The role of the coupling agent used in this study is similar to that of the functional ligands in Warshawsky and Upson's report, but, in a different manner. The difference lies in the chemical reaction of $\mathrm{Si}-\mathrm{OH}$ and $\mathrm{Pd}^{2+}$ to form the $\mathrm{Si}-\mathrm{O}-\mathrm{Pd}$ covalent bonding. This type of Si-O-M covalent bonding has been observed by other researchers in polymer coatings on metal substrates, and grafting polymerization with metal. ${ }^{19-21}$ However, in this study, this type of Si-O-M covalent bonding was applied in the reversed manner: metal coating on a submicron polymer spheres, and therefore, the submicron polymer spheres bear a "core-shell" structure (CSNP spheres (Figure 2)). The chemical reaction of nickel ion with hypophosphite was:

$$
\mathrm{Ni}^{2+}+2 \mathrm{H}_{2} \mathrm{PO}_{2}{ }^{-}+2 \mathrm{H}_{2} \mathrm{O} \rightarrow \mathrm{Ni}+2 \mathrm{H}_{3} \mathrm{PO}_{3}{ }^{-}+2 \mathrm{H}^{+}
$$

\section{FT-IR Characterization}

FT-IR can be used to identify the presence of functional groups in materials. Here, the spectrum was used to verify the presence of functional groups such as carboxyl $(\mathrm{COOH})$, trialkoxysilyl $\left(\mathrm{Si}(\mathrm{OR})_{3}\right)$ or trihydroxysilyl $\left(\mathrm{Si}(\mathrm{OH})_{3}\right)$ on the

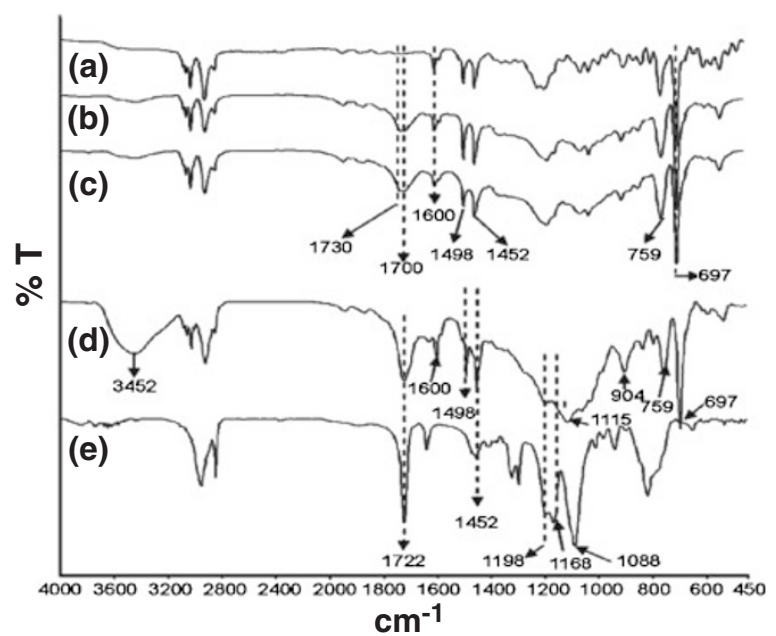

Figure 3. FT-IR spectra of submicron polymer spheres (a) $P(S t-D V B)$ (BC0), (b) $P($ St-DVB-AA) (BA-3), (c) P(St-DVB-MAA) (BM-3), (d) TSPM (3-trimethoxysilyl propryl methacrylate), (e) P(St-DVBTSPM) (BT-3).

sbumicron polymer spheres. Figure 3 compared the FT-IR spectra of submicron polymer spheres BA-3, BM-3, BT-3 and TSPM with the control (bare submicron polymer spheres BC$0)$. The characteristic absorption peaks of BC-0 showed a series of typical phenyl ring breathing absorptions at 1600, 1498 , and $1452 \mathrm{~cm}^{-1}$ while $3100-3000 \mathrm{~cm}^{-1}$ corresponds to the $\mathrm{C}-\mathrm{H}$ stretching of the phenyl ring, and the 758 and $697 \mathrm{~cm}^{-1}$ peaks correspond to the $\mathrm{C}-\mathrm{H}$ bending absorption of the phenyl ring. These absorption peaks verified the existence of phenyl ring (of styrene and DVB) as the major content of all the types of submicron polymer spheres under investigation.

The spectra of BA-3 and BM-3 submicron polymer spheres exhibited the characteristic carboxyl absorption peaks, in which $3600-3200 \mathrm{~cm}^{-1}$ corresponds to the $\mathrm{O}-\mathrm{H}$ of the carboxyl and, 1730 and $1700 \mathrm{~cm}^{-1}$ corresponds to the carbonyl $(\mathrm{C}=\mathrm{O})$ of the carboxyl. Together, they verified the copolymerization of AA

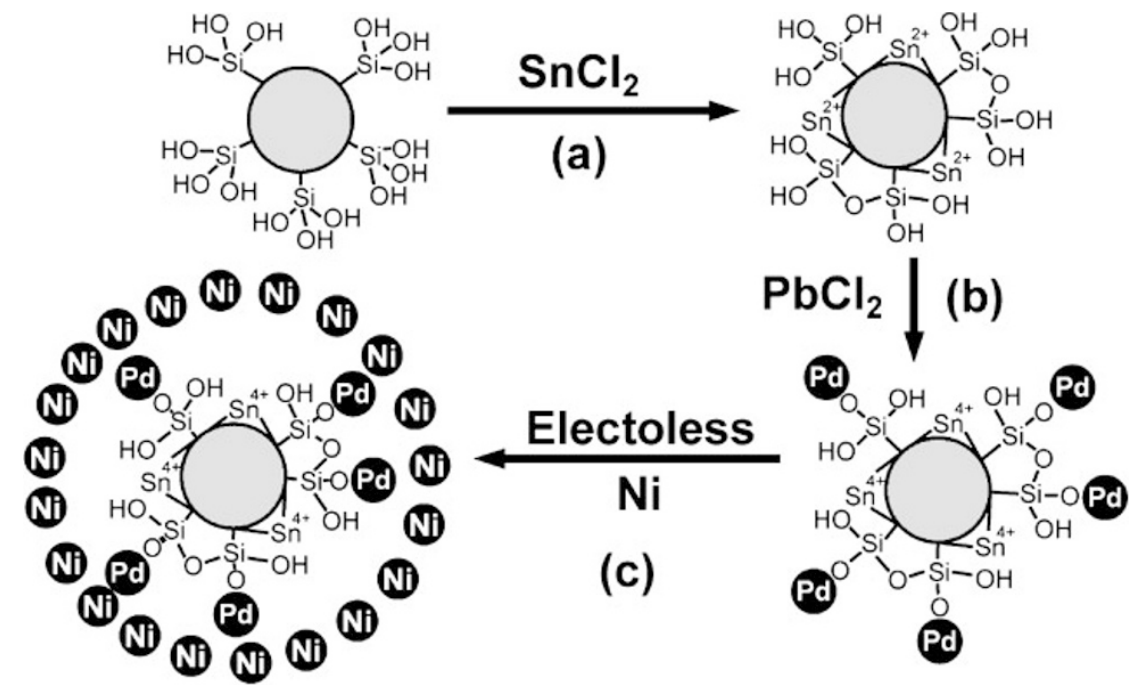

Figure 2. Schematic diagram of nickel electroless Ni-plating: (a) BT particles were pretreated by acid $\mathrm{SnCl}_{2} \mathrm{solution}$ (b) the $\mathrm{Sn}^{2+}$ treated $\mathrm{BT}$ particles were activated in $\mathrm{PdCl}_{2}$ solution; (c) electroless plating of $\mathrm{Ni}$. 
or MAA in the submicron polymer spheres (BA-3 and BM-3, in Figure 3 and Table I).

The spectrum of BT-3 submicron polymer spheres showed the characteristic broad absorption peaks of trihydroxysilyl group $\left(\mathrm{Si}(\mathrm{OH})_{3}\right)$ at $3600-3200 \mathrm{~cm}^{-1}(\mathrm{O}-\mathrm{H})$ and 1062 and $904 \mathrm{~cm}^{-1}(\mathrm{Si}-\mathrm{O}-\mathrm{H})$ and carbonyl group $(\mathrm{C}=\mathrm{O})$ at $1722 \mathrm{~cm}^{-1}$. In this BT-3 spectrum, only the absorption peaks of carbonyl group have the same wave number with that of TSPM $\left(1722 \mathrm{~cm}^{-1}\right)$. More evidence is required to be definitive about the formation of Si-O-H, and are discussed as follows.

During copolymerization between the monomers and the coupling agent, the silyl moiety of the coupling agent can take one of the three possible forms of covalent bonding: Si-O-C, $\mathrm{Si}-\mathrm{O}-\mathrm{Si}$ and $\mathrm{Si}-\mathrm{O}-\mathrm{H}$. The fist form belongs to the original trialkoxysilyl group. The second form results from the condensation reaction between the alkoxysilyl or hydroxysily groups. Alternatively, the third form may come from the hydrolysis of the alkoxysilyl group.

The bonding of $\mathrm{Si}-\mathrm{O}-\mathrm{C}, \mathrm{Si}-\mathrm{O}-\mathrm{Si}$ and $\mathrm{Si}-\mathrm{O}-\mathrm{H}$ all have absorption peaks in the range of 1100 to $1000 \mathrm{~cm}^{-1}$. It is not easy to differentiate between the three types of bonding solely by inspecting the spectra in Figure 3(d) and 3(e). However, for fixed number of trialkoxysilyl groups, increased formation of $\mathrm{Si}-\mathrm{O}-\mathrm{H}$ would relatively decrease the proportion of Si-O-C and Si-O-Si. The broad Si-O-H absorption peak $\left(3600-3200 \mathrm{~cm}^{-1}\right)$ in Figure 3(d) indicated significant portion of the trialkoxysilyl groups were hydrolyzed. Oppositely, if significant progress of condensation reaction was allowed to take place, the polymer latex would form aggregates, and formation of submicron polymer spheres would not be possible. However, such conclusion does not exclude the possibility of condensations between neighboring silyl groups forming $\mathrm{Si}-\mathrm{O}-\mathrm{Si}$.

Combining the above stated observations, it is fair to say that the hydrolysis of trialkoxysilyl group in the emulsion polymerization solution was the major change occured to the trihydroxysilyl groups during the polymerization reaction. The resulting $\mathrm{Si}-\mathrm{O}-\mathrm{H}$ function groups paved the way for subsequent covalent bonding with palladium in the surface activation step.

\section{XRD Analysis}

The presence of tin, palladium and nickel on the surface of the submicron polymer spheres were verified by XRD analysis. The XRD patterns of NiBA-3, NiBM-3 and NiBT-3 of the prepared CSNP spheres were shown in Figure 4. In the XRD profiles of these CSNP spheres, two strong reflection at $2 \theta=44^{\circ}$ and $52^{\circ}$ are attributed to the (111) and (200) planes of zero-valence nickel. ${ }^{22}$ This indicates that zero-valence nickel was successfully deposited on the surface of the submicron polymer spheres. Another strong reflection observed at $2 \theta=34^{\circ}$ and $40^{\circ}$ is attributed to the plane of tin (used in sensitization). ${ }^{23}$ A series of strong reflection at $2 \theta=40^{\circ}, 46^{\circ}$, and $66^{\circ}$ are attributed to the plane of zero valence-palladium. ${ }^{24}$ However, after verifying the presences of these metals, XRD did not provide understanding of how tightly is the metal layer bound to the polymer, and this is another issue that should be dealt with, which will be discussed shortly.

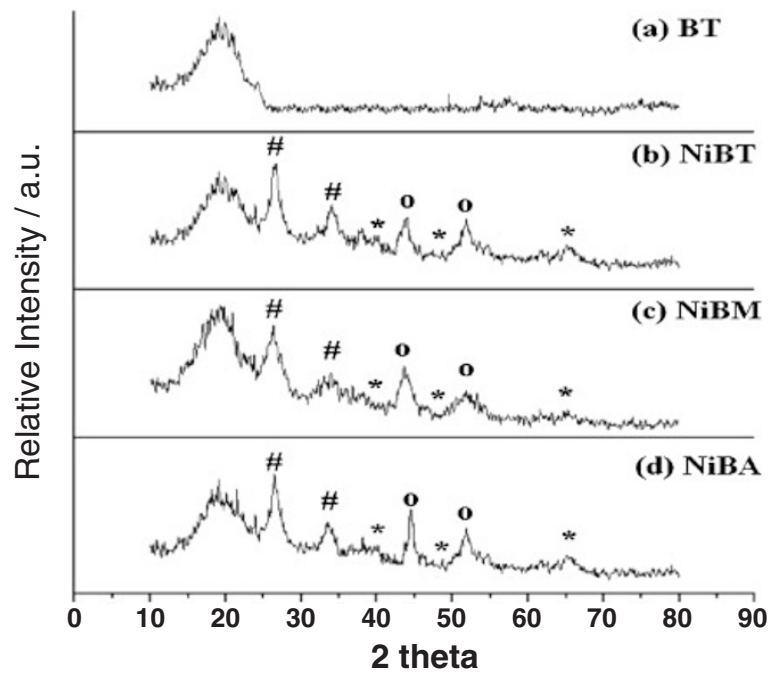

Figure 4. XRD curves of different submicron polymer spheres (a) BT-3, (b) NiBT-3, (c) NiBM-3, (d) NiBA-3 ( $*=\mathrm{Pd}$, \# = Sn, o = Ni).

\section{EDX Analysis}

EDX studies the energy level of the X-ray emitted when an electron beam strikes the surface of a material to identify elements contained in the material. The EDX spectra of submicron polymer spheres BT-3 and NiBT-3 of the CSNP spheres were shown in Figure 5. These spectra supported the results from XRD analysis stated above. The Si-peak in Figure 5(a) verified the presence of the coupling agent in the BT-3 polymer samples (without metal coating). The presence of sodium peak was due to the fact that during the emulsifierfree emulsion polymerization reaction, sodium hydroxide was used to maintain a basic environment such that AA and MAA are mostly deprotonated and thus can function as a reactive self-emulsifier. According to Macaskie et al. ${ }^{25}$ and Bonnemann et $a l .,{ }^{26}$ the palladium peak should be observed at $2.8-2.9 \mathrm{keV}$ in Figure 5(b), but instead, the peak at that position was labeled as Sn. It is quite likely that the peaks of Sn (at 2.9, 3.4 and $4.3 \mathrm{keV}$ ) were too close to the palladium peak, and partially overlapped with each other, and the latter was left unlabeled by the instrument.

\section{FESEM Analysis}

The FESEM micrographs can be used to study the morphology and microscopic structure of materials. Typical FESEM micrographs of a single particle of the CSNP spheres: NiBT-3, NiBM-3 and NiBA-3 are shown in Figure 6(a), 6(b) and 6(c) respectively. Three distinctly different particle sizes can be observed in Figure 6 (335, 311 and $233 \mathrm{~nm}$ for NiBT-3, NiBM-3 and NiBA-3; Table I).

In an emulsion polymerization system, increased concentration of emulsifier will lead to smaller particle size. For this reason, in the emulsifier-free emulsion polymerization system, with the presence of $\mathrm{NaOH}$, the carboxyl groups of methacrylic acid (MAA) in NiBM-3 and acrylic acid (AA) in NiBA-3 both tend to form sodium salts and function as self-reactive emulsifier. The molecular weight of MAA (NiBM-3), with an 

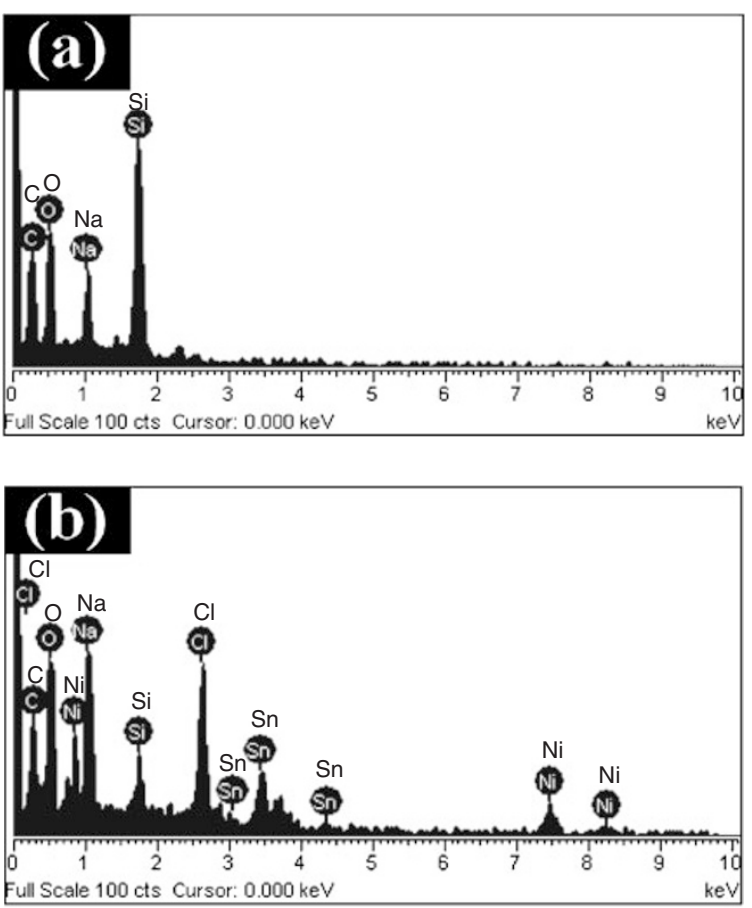

Figure 5. EDX images of (a) BT-3 and (b) NiBT-3.
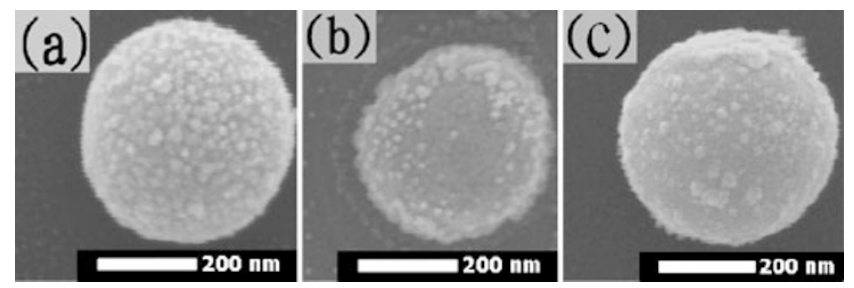

Figure 6. FESEM images of a CSNP sphere: (a) NiBT-3, (b) NiBA-3, and (c) NiBM-3.

extra methyl group (on vinyl carbon of AA), is higher than that of AA (NiBA-3). Thus, for equal weight of the two, the former has less number of molecules than the latter, which means more amount of emulsifier in the case of NiBA-3. This leads to the result that the particle size of NiBA-3 turned out to be smaller than that of NiBM-3 (for the same wt \% of AA and MAA, Table I). In case of NiBT, on the other hand, the acidity of $\mathrm{Si}-\mathrm{OH}$ was much lower than that of $\mathrm{COOH}$, and is far less hydrophilic, which in turn makes it lack of emulsifying ability. Consequently, the particle sizes of NiBM-3 and NiBA-3 are smaller than that of NiBT.

Another implication worth mentioning in Figure 6 is that the plated nickel on NiBT-3 is richer and more uniformly distributed on the surface of NiBT-3 than the other two samples. Such contrast is further supported by both weight residue and conductivity measurements as shown in Figures 10 and 12, which will be discussed later. This phenomenon could be attributed to the fact that every $\mathrm{Si}$-atom on each coupling agent molecule has three available $\mathrm{Si}-\mathrm{O}-\mathrm{H}$ groups for forming the palladium catalytic sites. Although for each $\mathrm{Si}$ atom, the number of bonded palladium atom is likely to be less than three

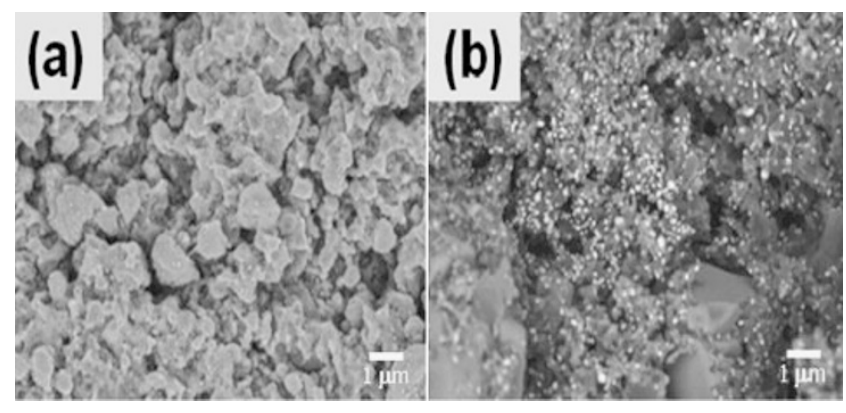

Figure 7. BEl images of (a) BT-3 $(x 10,000)$ and (b) NiBT-3 $(\times 10,000)$ after TGA.

due to inherent spatial interference, more opportunity and stability of bonding with palladium are expected, and which is one of the advantages of employing the coupling agent proposed in this study.

Besides bonding with palladium atom, one or two of the three $\mathrm{Si}-\mathrm{O}-\mathrm{H}$ groups may contribute, in another way, to the formation of stable and strong bridge between the polymer core and the metal shell. The neighboring $\mathrm{C}-\mathrm{Si}-(\mathrm{OH})_{3}$ groups (Figure 2), on the surface of the polymer core, may form lateral $\mathrm{Si}-\mathrm{O}-\mathrm{Si}$ covalent bonds by condensation reaction. Such $\mathrm{Si}-\mathrm{O}-\mathrm{Si}$ covalent bonds provided a two dimensional network on the surface of the polymer core. Consequently such two dimensional network further stabilized the interface between the polymer core and the metal shell.

Figure 7 compares the backscattered electron image (BEI) photographs of BT-3 and NiBT-3 after sintering $\left(800^{\circ} \mathrm{C}\right)$ for thermal gravimetric analysis (TGA). Figure 7(a) shows that after TGA sintering, the BT-3 has lost their integrity as spheres. In Figure 7(b), among the debris of polymer spheres, there are non-penetrated nickel particles shown as white dots. This shows that these nanosized nickel particles were plated on the surface of the CSNP spheres. The sizes of these nickel particles were approximately $20 \mathrm{~nm}$ as shown in Figure 6(a).

\section{TEM Analysis}

Thickness of the nickel plating layer is another variable to be controlled in the design of CSNP spheres. In order to study the influence of the concentration of electroless chemical plating solution on the thickness of the plated nickel layer, two different concentrations of electroless chemical plating solution (ENP-1 and ENP-2, Table II) were used on the same load of the submicron polymer spheres, for preparing the NiBT series CSNP spheres. TEM was used to observe variation of the thickness of the deposited nickel shell when the concentrations of the key components in the plating solution were doubled (Table II).

Figure 8 compares the thicknesses of the "nickel shell" by TEM micrographs of NiBT-3 CSNP spheres. One important feature Figure 8 shows is that the dark rims surround the spheres (the "nickel shell") are continuous and are rather uniform. The average thicknesses can be estimated by comparing the thicknesses to the scale bar. The nickel shell for CSNP spheres prepared from ENP-2 electroless chemical 
(a)

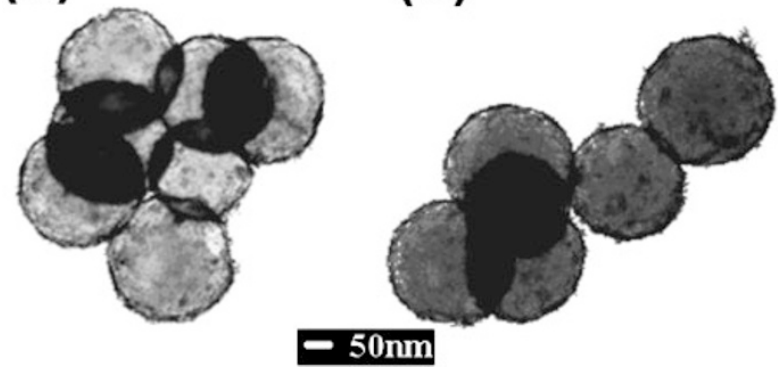

Figure 8. TEM images of the NiBT-3 CSNP spheres from different concentrations of electroless plating solution: (a) ENP-1and (b) ENP-2.

deposition solutions were thicker than those prepared from ENP-1 (Table II).

Another important feature in Figure 8 is the different electron penetrability observed for the CSNP spheres prepared from electroless chemical deposition solutions of different nickel concentrations. The darker image shown in Figure 8(b) indicated lower electron penetrability, and therefore, thicker nickel layer. The concentration of the electroless chemical deposition solution can serve as a design variable in preparing the CSNP spheres with desirable nickel thickness.

\section{Thermal Properties}

In analyzing the thermal properties of materials, there are several methods commonly adopted, and each provides different physical aspects. In this research, the thermal degradation temperature ( $T_{\mathrm{d} 5}$, temperature at $5 \%$ weight loss) and weight residues of the BT series submicron polymer spheres and, the nickel plated NiBA-3, NiBM-3 and NiBT series of the prepared CSNP spheres were studied.

The thermal stability of a polymer material is strongly dependent on the strength of the covalent bonds between the atoms that form the polymer molecules. The bond dissociation energy of $\mathrm{C}-\mathrm{C}$ bond is lower than that of $\mathrm{C}-\mathrm{Si}$ and $\mathrm{Si}-\mathrm{O}$ bond. Based on this, it is expected that for the prepared BT series submicron polymer spheres, the thermal degradation temperature should increase with increasing TSPM content. Figure 9 shows that the $T_{\mathrm{d} 5} \mathrm{~S}$ of the $\mathrm{BT}$ series submicron polymer spheres, with TSPM content of 1, 2, 3, 4 and 5\%, were 377 , $379,381,386$, and $387^{\circ} \mathrm{C}$. This trend is in accordance with the amount of TSPM, and hence Si-O-Si network formed by condensation between neighboring $\mathrm{Si}-\mathrm{OH}$ on the surface of the polymer core. However, the dosage of TSPM was low (1-5\%), and its influence on $T_{\mathrm{d} 5}$ was minor.

Figure 10 compares the TGA curves of NiBA-3, NiBM-3 and NiBT-3 CSNP spheres. The weight residues of NiBA-3, NiBM-3 and NiBT-3 were 17, 18 and 23\% respectively. Also, for comparison, the TGA curve of BT-3 was put in Figure 10. The weight residues of BT-3, in Figure 10, was only $4 \%$ (values for BA-3 and BM-3 were approximately the same, data not shown).

Knowing that among the metals on the polymer spheres' surfaces, nickel is the major one, while palladium and tin

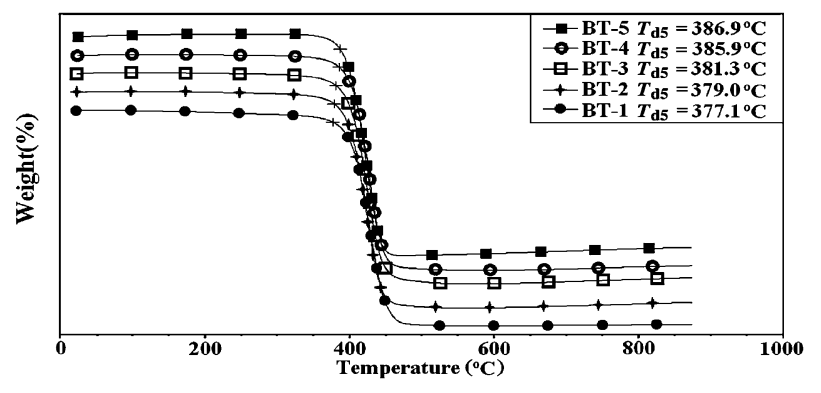

Figure 9. TGA curves of the submicron polymer spheres with different TSPM content (BT-1 to BT-5) ( $T_{\mathrm{d} 5}$, temperature at $5 \%$ weight loss).

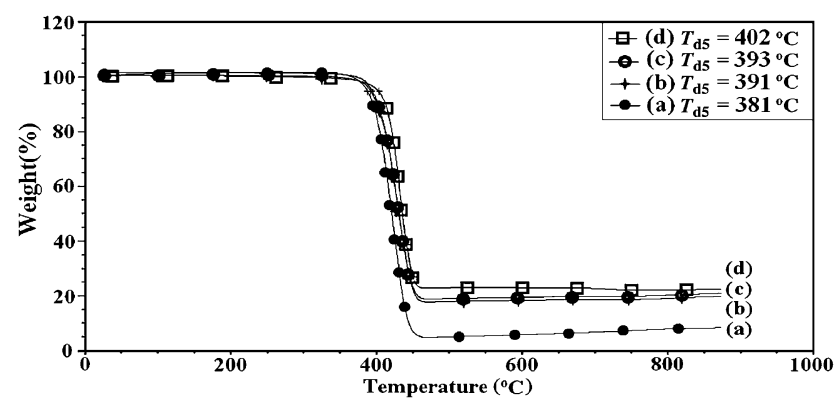

Figure 10. TGA curves of different CSNP spheres (a) BT-3, (b) NiBA-3, (c) NiBM-3, (d) NiBT-3 ( $T_{\mathrm{d} 5}$, temperature at $5 \%$ weight loss).

contents are relatively minor, and the differences between the weight residues of the CSNP spheres and that of the bare polymers should mainly be attributed to nickel content. Then, larger difference in weight residues (between the CSNP spheres and the bare submicron polymer spheres) for NiBT-3 (23\%) and BT-3 (4\%), than those of NiBA-3 and NiBM-3 would indicate higher nickel content in NiBT-3. Such difference could account for $5-6 \%$ (of the original weight) more nickel that was plated on NiBT-3, than on NiBA-3 and NiBM-3.

Figure 10 also provided comparisons of the $T_{\mathrm{d} 5} \mathrm{~s}$ of NiBA-3, NiBM-3 and NiBT-3, which were 391,393 and $402{ }^{\circ} \mathrm{C}$ respectively. This trend is the same as that of the weight residues. In that the $T_{\mathrm{d}}$ of NiBT-3 was higher than the other two, while the difference between $T_{\mathrm{d} 5} \mathrm{~S}$ of NiBA-3 and NiBM-3 was minor.

\section{Mechanical Stability}

The binding strength between metal and polymer, and the firmness of the polymer per se, are both important chemical qualities to be understood before potential applications are feasible. Good mechanical stability is especially important in preparing conductive coatings or adhesives, since the process commonly involves ball mill grinding for fine dispersion of the metallic filler in the polymer solution. Studying the mechanical stability in ball mill is adopted for this purpose. The three types of CSNP spheres (NiBA-3, NiBM-3 and NiBT-3) were subject to ball mill for different time lengths $(0.5,1.0$ and $2.0 \mathrm{~h})$ and compared the results in terms of FESEM micrographs and conductivities in solutions (discussed in the next subsection). 

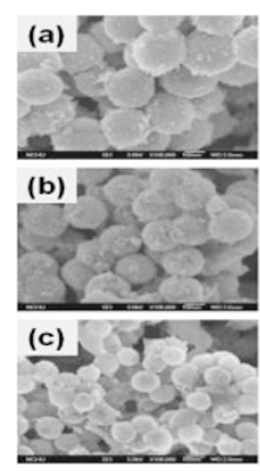

$(0.5 \mathrm{~h})$
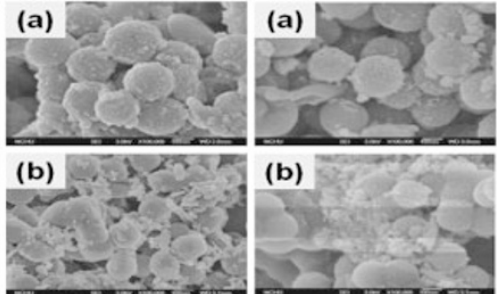

(b)

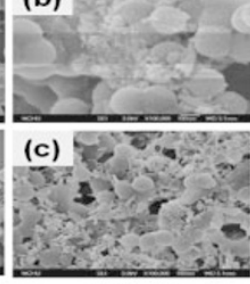

$(2.0 \mathrm{~h})$
Figure 11. FESEM images $(\times 100,000)$ of the CSNP spheres after ball mill of 0.5, 1.0 and 2.0 h: (a) NiBT-3, (b) NiBM-3, (c) NiBA-3.

Figure 11 compared the FESEM micrographs of the NiBA-3, NiBM-3 and NiBT-3 CSNP spheres after ball mill treatment. After $0.5 \mathrm{~h}$ of ball milling, all three samples remained virtually intact. After $1.0 \mathrm{~h}$ of milling, NiBT-3 remained intact, while NiBA-3 and NiBM-3 were not able to maintain their integrities. When milling time was further extended to $2.0 \mathrm{~h}$, the damage to NiBT-3 was minor as compared to NiBA-3 and NiBM-3. The coupling agent seemed to provide stronger bondage between the metal shell and the polymer core and led to better mechanical stability, than the other two (NiBA-3 and NiBM-3), and made it more durable in ball milling. Meanwhile, although the nickel coated surfaces of NiBT-3 did not look to be severely harmed after $2.0 \mathrm{~h}$ of ball milling, measuring the conductivity in solution (to be discussed in the next subsection) should be a better criterion of whether the metal layer, after ball mill, remained firmly attached to the polymer matrix.

The above observations, for NiBT-3, showing better mechanical stability, thermal properties, as well as denser and more uniformly plated nickel could also be explained by the difference in bonding natures between the two types of functional ligands incorporated (-Si-OH or $-\mathrm{COOH})$ with palladium. Since the acidity of $\mathrm{Si}-\mathrm{O}-\mathrm{H}$ is much lower than $\mathrm{COOH}$, the latter tends more to form a salt with metal. Therefore the COO-Pd bonding is more likely to be ionic or coordination bonding. On the other hand, Si-O-Pd is more likely to be covalent bonding, since Si-O$\mathrm{H}$ does not form salt with metals. Consequently, the more stable Si-O-Pd covalent bonding resulted in a stronger bondage between the metal and the polymer. Meanwhile, as mentioned above that there are, for each $\mathrm{Si}$ atom, three -OH groups to provide more opportunity for binding palladium. Also, as discussed above, the two dimension Si-O-Si network could well be another reason for improved mechanical stability. The combination of the two reasons is quite likely to be the cause leading to a denser and more uniformly distributed nickel coating on the CSNP spheres' surfaces (Figure 6).

\section{Conductivity of Aqueous Suspension of CSNP Spheres}

For the CSNP spheres to be useful in the field of conductive ink, coating or adhesives, the material should have good electrical conductivity in liquid suspension. Figure 12 compares

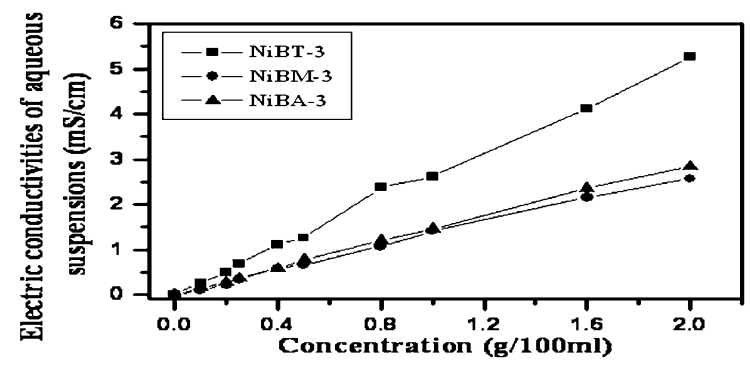

Figure 12. Electric conductivities of aqueous $(\mathrm{pH} 6)$ suspensions of NiBT-3, NiBM-3, NiBA-3 CSNP spheres at various concentrations.

the electrical conductivities, of NiBT-3, NiBM-3 and NiBA-3 suspended at various concentration in water solutions at $\mathrm{pH} 6$. Figure 12 shows that the aqueous suspensions' conductivities all increased linearly with increasing concentrations of the CSNP spheres. For the same concentration, the NiBT-3 suspension had the highest aqueous conductivity, while that of the other two (NiBM-3 and NiBA-3) were much lower. This observation could be attributed to the use of the coupling agent which provided more opportunity and stronger binding for nickel.

Frequently, for such type of materials to be applicable in a conductive ink, prior to dispersion in resin solution, the powder may need to be ball milled either for reduction of particle size or for separation of aggregated powder for better dispersion in the solution. Therefore, electrical conductivity after ball mill is an important index of the quality of the CSNP spheres.

Figure 13 compares the suspensions' electrical conductivities, as a function of concentration and time length of ball milling. All three samples (NiBT-3, NiBM-3 and NiBA-3) lost electrical conductivity after $8 \mathrm{~h}$ of ball milling. For milling time shorter than $1 \mathrm{~h}$, NiBT-3 (Figure 13(a)) demonstrated superior ability to sustain mechanical forces as compared to that of NiBM-3 (Figure 13(b)) and NiBA-3 (Figure 13(c)). Figure 13 shows that for the same concentration of $2.0 \mathrm{~g} / 100 \mathrm{~mL}$, after $1 \mathrm{~h}$ of ball milling, for NiBT-3, the liquid conductivity dropped only $14 \%$ (from 5.25 to $4.50 \mathrm{mS} / \mathrm{cm}$ ), while that for NiBM-3 and NiBA-3 were $44 \%$ (from 2.56 to $1.42 \mathrm{mS} / \mathrm{cm}$ ) and $38 \%$ (from 2.75 to $2.00 \mathrm{mS} / \mathrm{cm}$ ) respectively. This indicates that the coupling agent successfully provided strong bondage between the polymer core and the metal shell, which is also observed in the FESEM micrographs (Figure 11).

Parallel to these observations stated above, Figure 14 shows that the rate of loss of electric conductivity on milling time, the slope, of NiBT-3 is much lower than that of NiBM-3 and NiBA-3, especially for milling times shorter than $1 \mathrm{~h}$. This reflects that the bondage between the polymer core and the metal shell was weaker for NiBM-3 and NiBA-3 than for NiBT-3, such that the former two lose conductivity, during milling, at a rate faster than NiBT-3.

In the above stated measurements for electric conductivities of aqueous suspension of the CSNP spheres, the intention was to use it as an indication of the strength of the bondage between the Ni-shell and the polymer core. The intrinsic electric conductivities of the CSNP spheres could not be obtained from these data and need to be further investigated. 

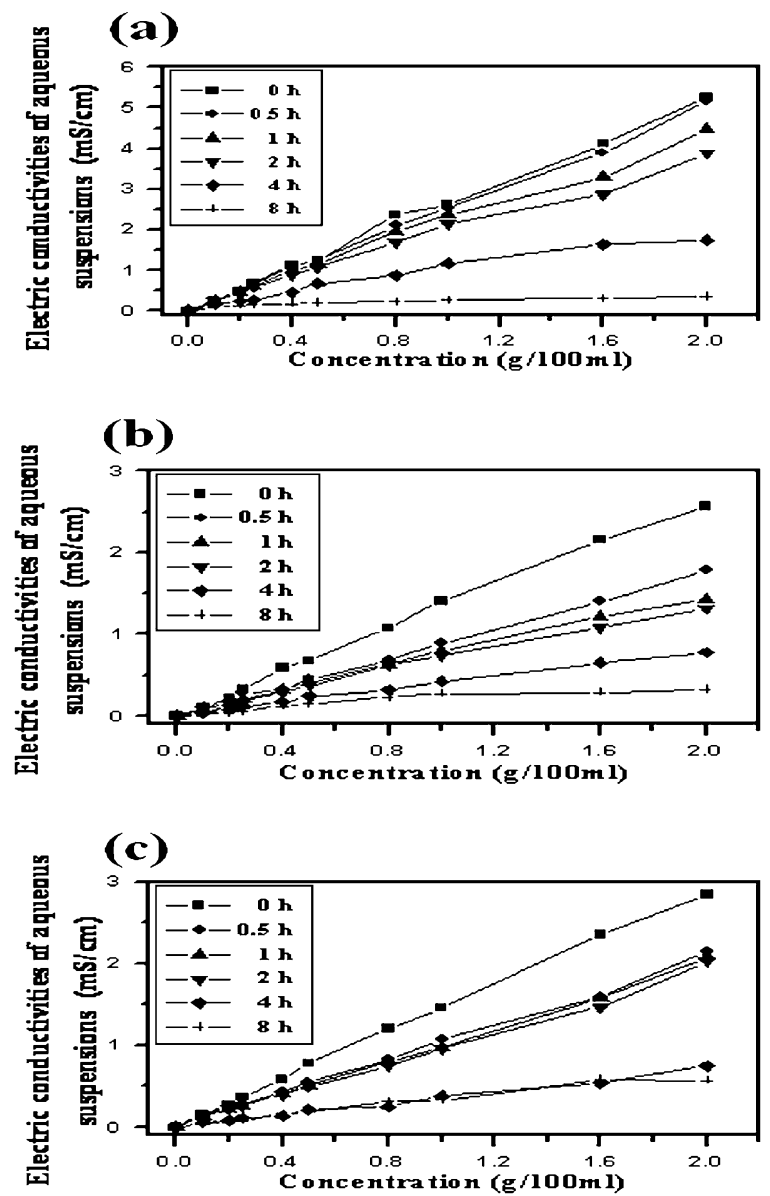

Figure 13. Electric conductivities of aqueous $(\mathrm{pH} 6)$ suspensions of CSNP spheres after ball mill of $0,0.5,1,2,4$, and $8 \mathrm{~h}$ : (a) NiBT-3, (b) NiBM-3, (c) NiBA-3.

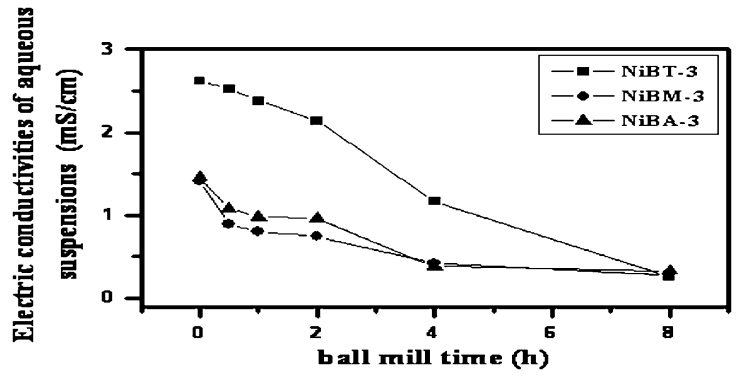

Figure 14. Electric conductivities of aqueous $(\mathrm{pH} 6)$ suspensions of NiBT-3, NiBM-3, NiBA-3 CSNP spheres after ball mill of $0,0.5,1,2,4$, and $8 \mathrm{~h}$, solid concentration was $2 \mathrm{~g} / 100 \mathrm{~mL}$.

\section{CONCLUSIONS}

Three series of submicron polymer spheres were synthesized by emulsifier free emulsion polymerization with the functional monomers, AA and MAA as well as the silane coupling agent, TSPM. The silane bridged electroless nickel plating on submicron polymer spheres were proven to provide strong bondage between the polymer core and the metal shell. The silane bridge at the polymer/metal interface led to a denser and more uniform distribution of metal coating, as well as enhanced mechanical and thermal stabilities. The silane bridged CSNP sphere produced (before and after ball milling) in this study was demonstrated to have superior electrical conductivity in aqueous suspension.

Received: December 26, 2008 Accepted: April 23, 2009 Published: June 17, 2009

\section{REFERENCES}

1. N. Bicak, S. Sungur, N. Tan, F. Bensebaa, and Y. Deslandes, J. Polym. Sci., Part A: Polym. Chem., 40, 748 (2002).

2. Y. Wang, Y. Wang, and L. Feng, J. Appl. Polym. Sci., 64, 1843 (1997).

3. H. X. Guo, Z. P. Qin, J. Wei, and C. X. Qin, Surf. Coat. Technol., 200, 2531 (2005).

4. E. Kandare, H. Deng, D. Wang, and J. M. Hossenlopp, Polym. Adv. Technol., 17, 312 (2006).

5. H. Ni, G. Ma, M. Nagai, and S. Omi, J. Appl. Polym. Sci., 80, 1988 (2001).

6. J. B. Jun, M. S. Seo, S. H. Cho, J. G. Park, J. H. Ryu, and K. D. Suh, J. Appl. Polym. Sci., 100, 3801 (2006).

7. A. Tuncel, H. Çiçek, M. Hayran, and E. Pişkin, J. Biomed. Mater. Res., 29, 721 (1995).

8. S. Jiang, E. D. Sudol, V. L. Dimonie, and M. S. El-Aasser, J. Appl. Polym. Sci., 108, 4096 (2008).

9. M. Cao, B. Tong, J. Shen, Y. Dong, and J. Zhi, J. Appl. Polym. Sci., 109, 1189 (2008).

10. J. Zhang, X. Li, S. Chai, S. Wang, and S. Cheng, J. Appl. Polym. Sci., 97, 1681 (2005).

11. H. Fukumoto, Y. Muramatsu, T. Yamamoto, J. Yamaguchi, K. Itaka, and H. Koinuma, Macromol. Rapid Commun., 25, 196 (2004).

12. A. Cuña, A. Noguera, E. Saucedo, and L. Fornaro, Cryst. Res. Technol., 39, 912 (2004).

13. W. C. Wang, R. K. H. Vora, E. T. Kang, and K. G. Neoh, Macromol. Mater. Eng., 288, 152 (2003).

14. T. Sterzynski and H. Øysæd, Polym. Eng. Sci., 44, 352 (2004).

15. D. Wu, T. Zhang, W. C. Wang, L. Zhang, and R. Jin, Polym. Adv. Technol., 19, 335 (2008).

16. R. Shirahata, M. Suzuki, Y. Yamada, T. Kitamoto, and S. Dezawa, U. S. Patent 4089993 (1978).

17. A. Warshawsky and D. A. Upson, J. Polym. Sci., Part A: Polym. Chem., 27, 2963 (1989).

18. J. G. Park, J. W. Kim, S. Oh, and K. D. Suh, J. Appl. Polym. Sci., 87, 420 (2003).

19. M. Z. Rong, Q. L. Ji, M. Q. Zhang, and K. Friedrich, Eur. Polym. J., 38, 1573 (2002).

20. V. Smuleac, D. A. Butterfield, S. K. Sikdar, R. S. Varma, and D. Bhattacharyya, J. Membrane. Sci., 251, 169 (2005).

21. L. Wang and J. Sheng, J. Macromol. Sci., Part A: Pure Appl. Chem., 40, 1135 (2003).

22. F. Tao, Y. Liang, G. Yin, D. Xu, Z. Jiang, H. Li, M. Han, Y. Song, Z. Xie, Z. Xue, J. Zhu, Z. Xu, L. Zheng, X. Wei, and Y. Ni, Adv. Funct. Mater., 17, 1124 (2007).

23. N. Rezlescu, C. Doroftei, E. Rezlescu, and P. D. Popa, Phys. Status Solidi., 203, 306 (2006).

24. V. I. Pârvulescu, V. Pârvulescu, U. Endruschat, G. Filoti, F. E. Wagner, C. Kübel, and R. Richards, Chem. Eur. J., 12, 2343 (2006).

25. L. E. Macaskie, N. J. Creamer, A. M. M. Essa, and N. L. Brown, Biotechnol. Bioeng., 96, 631 (2007).

26. H. Bönnemann, W. Brijoux, K. Siepen, J. Hormes, R. Franke, J. Pollmann, and J. Rothe, Appl. Organomet. Chem., 11, 783 (1997). 